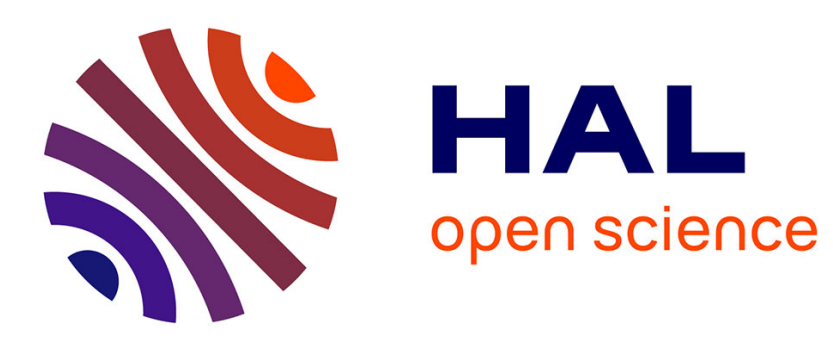

\title{
Molecular dynamics modeling of helium bubbles in austenitic steels
}

\author{
A. Jelea
}

\section{To cite this version:}

A. Jelea. Molecular dynamics modeling of helium bubbles in austenitic steels. Nuclear Instruments and Methods in Physics Research Section B: Beam Interactions with Materials and Atoms, 2018, 425, pp.50-54. 10.1016/j.nimb.2018.04.008 . hal-02874503

\section{HAL Id: hal-02874503 https://hal.science/hal-02874503}

Submitted on 16 Jul 2020

HAL is a multi-disciplinary open access archive for the deposit and dissemination of scientific research documents, whether they are published or not. The documents may come from teaching and research institutions in France or abroad, or from public or private research centers.
L'archive ouverte pluridisciplinaire HAL, est destinée au dépôt et à la diffusion de documents scientifiques de niveau recherche, publiés ou non, émanant des établissements d'enseignement et de recherche français ou étrangers, des laboratoires publics ou privés. 


\title{
Molecular dynamics modeling of helium bubbles in austenitic steels
}

\author{
A. Jelea* \\ Institut de Radioprotection et de Sûreté Nucléaire (IRSN), PSN/SEMIA/LPTM, BP3, 13115 \\ Saint-Paul-lez-Durance, France
}

\begin{abstract}
The austenitic steel devices from pressurized water reactors are continuously subjected to neutron irradiation that produces crystalline point defects and helium atoms in the steel matrix. These species evolve into large defects such as dislocation loops and helium filled bubbles. This paper analyzes, through molecular dynamics simulations with recently developed interatomic potentials, the impact of the helium/steel interface on the helium behavior in nanosize bubbles trapped in an austenitic steel matrix. It is shown that the repulsive helium-steel interactions induce higher pressures in the bubble compared to bulk helium at the same temperature and average density. A new equation of state for helium is proposed in order to take into account these interface effects.
\end{abstract}

Keywords: helium bubble, austenitic steel, molecular dynamics

Corresponding author at: Institut de Radioprotection et de Sûreté Nucléaire (IRSN), PSN/SEMIA/LPTM, BP3, 13115 Saint-Paul-lez-Durance, France

E-mail address: andrei.jelea@irsn.fr (A. Jelea) 


\section{Introduction}

Austenitic steels are used in the Pressurized Water Reactors (PWR) as materials for the internal components. They are preferred due to their good mechanical properties and their high resistance to corrosion. Some of the most common types of austenitic steels, AISI-304 and AISI-316, are alloys of iron (Fe), chromium (Cr) and nickel (Ni) with small amounts of other elements, such as manganese (Mn), molybdenum (Mo), silicon (Si) and carbon (C) [1]. During their lifetime in the nuclear reactor, the austenitic steel devices are subjected to neutron irradiation at elevated temperature. The collisions between high energy neutrons and steel atoms produce point defects (vacancies and self-interstitial atoms) that can evolve into large clusters of defects such as voids (clusters of vacancies) or dislocation loops (clusters of self-interstitial atoms or vacancies). Transmutation reactions of $(n, \alpha)$ type take place between neutrons and alloy atoms (especially Ni atoms) [2]. The main products of these reactions are helium (He) and hydrogen (H). Small quantities of these two elements can have a strong impact on steel properties, especially the mechanical ones [2].

The helium atoms act as a vacancy trap forming helium-vacancy complexes (helium bubbles) [3,4]. Experimental studies on helium implanted samples of austenitic steels [5-13] revealed the existence of small, mainly spherical, bubbles with diameters ranging from less than a nanometer [7] to more than a hundred of nanometers [6]. The helium bubbles number density in the steel matrix varies from $10^{20} \mathrm{~m}^{-3}$ to $10^{24} \mathrm{~m}^{-3}$. The bubbles average size and number density are strongly depending on the steel temperature and the fluence of implantation [12].

Several theoretical studies have been devoted to understanding the formation of helium bubbles in body-centered cubic (bcc) iron or iron-chromium and the behaviour of helium in these bubbles [14-23]. As the relationship between the average helium density and the 
pressure in the bubble was found to be strongly altered with respect to bulk helium, new equations of state that take into account the helium/iron interface effects have been proposed for helium in nanobubbles [22,23].

The recent development of a ternary FeNiCr potential [24] opened the door to extending the atomistic studies to helium bubbles in metal alloys similar in structure and composition to some austenitic steels that are frequently used in the nuclear industry. The present work is a molecular dynamics study on the helium behaviour in nanosize bubbles in a face centred cubic (fcc) FeNiCr alloy at compositions similar to AISI-316 austenitic steels. We propose a simple equation of state (EOS) for helium in nanobubbles that takes into account the helium/steel interface effects. This equation is conceived to reasonably describe the helium in nanobubbles for helium densities in agreement with experimental observations and temperature-pressure conditions similar to those in the PWRs.

The paper is structured as follows: Section 2 details the models and methods employed in this study, Section 3 presents the steps taken to build the equation of state for helium in nanobubbles and the results of this study are summarized in Section 4.

\section{Models and methods}

\subsection{Models for helium bubbles in steel}

All the calculations presented here were carried out using 3D periodic boundary conditions. The pattern for the periodical model was built starting from a supercell of $15 \times 15$ x 15 fcc elementary cells. The fcc sites of this supercell were randomly filled with $\mathrm{Fe}$, Ni and Cr atoms in order to satisfy two conditions: 
- The alloy should exhibit an homogeneous distribution of its components (Fe, $\mathrm{Ni}, \mathrm{Cr}$ ) [25];

- The alloy composition (in weight $\%$ ) should be: $12 \% \mathrm{Ni}, 17.5 \% \mathrm{Cr}$ and $70.5 \% \mathrm{Fe}$. This composition is similar to AISI-316, but also to some AISI-304 alloys [1].

The resulting system was a perfect fcc supercell, with the cell parameter $a=5.325 \mathrm{~nm}$, containing $1530 \mathrm{Ni}$ atoms, $2520 \mathrm{Cr}$ atoms and $9450 \mathrm{Fe}$ atoms. Therefore, the composition of the model system in $\mathrm{Ni}, \mathrm{Cr}$ and $\mathrm{Fe}$ is 11.3 atomic $\%, 18.7$ atomic $\%$ and 70 atomic \%, respectively.

Cavities with different $R_{C}$ radii $\left(R_{C}=0.5 \mathrm{~nm}\right.$ to $\left.1.5 \mathrm{~nm}\right)$ were carved by eliminating metal atoms in the middle of this supercell. The cavities were then filled with different numbers of helium atoms in order to cover a range of helium densities, from $20 \mathrm{~nm}^{-3}$ to $100 \mathrm{~nm}^{-3}$. This helium density range is in agreement with the experimental findings. Indeed, an Electron Energy-Loss Spectroscopy (EELS) study of helium bubbles in a FeCr martensitic steel [26] found $\mathrm{He}$ densities ranging from $20 \mathrm{~nm}^{-3}$ (5 nm radius bubbles) to $70 \mathrm{~nm}^{-3}$ (2 nm radius bubbles). Another EELS study on a FeCr ferritic alloy [27] found a He density of $61.3 \mathrm{~nm}^{-3}$ in bubbles with radii of about $1.4 \mathrm{~nm}$. Finally, a Positron Annihilation Spectroscopy (PAS) study [13], carried out on an austenitic steel of type 316, found a helium density of $56 \mathrm{~nm}^{-3}$ in bubbles with radii of about $4 \mathrm{~nm}$.

Previous theoretical and experimental works showed that the gas density in bubbles trapped in a solid matrix cannot surpass a certain value. Beyond this density limit, the bubble volume increases by surface breaking, in the sense that some solid atoms on the bubble surface are pushed into the interstitial sites of the matrix $[28,29]$. This phenomenon leads to distortions at the solid surface. The surface breaking is followed by a phenomenon of 
emission of an interstitial dislocation loop (known under the name of loop punch-out mechanism) $[29,30]$ that partially restores the perfect solid surface [17]. In the particular case of helium bubbles in perfect bcc $\mathrm{Fe}$ and $\mathrm{FeCr}$ alloys, the density limit value for which surface breaking occurs was estimated at $2 \mathrm{He} / \mathrm{Vac}\left(\sim 170 \mathrm{~nm}^{-3}\right)[15,17]$. However, the alloys under irradiation conditions contain a variable amount of vacancies that absorb at the bubble surface in order to establish the equality of their chemical potential at the bubble surface and in the bulk [21]. The vacancy absorption lowers the density limit at which the surface relaxation takes place. This could explain the fact that the experimental helium densities [26,27] are considerably lower than the theoretical limit estimated for these very systems $[15,17]$.

\subsection{Simulation methods}

The systems created as previously described were relaxed through NPT (constant Number of particles, Pressure and Temperature) molecular dynamics (MD) methods [31] as implemented in the LAMMPS code [32].

The MD simulations were carried out at $500 \mathrm{~K}$ and $700 \mathrm{~K}$ for an external pressure of 0 GPa. This $T-P$ range is very similar with the PWR conditions: temperature from $559 \mathrm{~K}$ to 603 $\mathrm{K}$ and a relatively low pressure of $0.0155 \mathrm{GPa}$.

A timestep of 0.2 fs was proved to be appropriate for this type of simulations [22]. The systems were relaxed during $1 \mathrm{~ns}\left(5 \times 10^{6} \mathrm{MD}\right.$ steps), then the results were accumulated over the next $5 \times 10^{6}$ MD steps at every 100 steps. Thus, for every case, one obtained a set of 50000 instantaneous values. The properties of interest (pressure in the bubble, density and bubble radius) were then calculated as averages of these instantaneous values.

The pressure in the bubble was computed from the atomic stress tensor diagonal 
components (provided by LAMMPS in pressure*volume units [32]) of the helium atoms contained in the bubble and the bubble volume. The bubble volume was that of a sphere with the radius $\left(R_{B}\right)$ being calculated as the arithmetic mean between a "cavity minimum radius" and a "cluster maximum radius". The "cavity minimum radius" was defined as the distance between the bubble mass center and the closest matrix atom (Fe, $\mathrm{Ni}$ or $\mathrm{Cr}$ ) and the "cluster maximum radius" as the distance between the bubble mass center and the furthest (helium) atom in the helium cluster. Following this definition [33], the bubble/steel geometrical interface would be situated at the crossing point of the matter densities of the two phases.

\subsection{Interatomic potentials}

All the simulations were performed using semi empirical interatomic potentials to describe the three types of interactions that are present in the systems: the Fe-Ni-Cr interactions, the $\mathrm{M}-\mathrm{He}(\mathrm{M}=\mathrm{Fe}, \mathrm{Ni}, \mathrm{Cr})$ interactions and the He-He interactions.

For the Fe-Ni-Cr interactions one used a ternary embedded atom method (EAM) type potential [24]. This potential is able to well reproduce, with respect to Density Functional Theory (DFT) and experimental results, the stability of the fcc phase, the elastic constants and the stacking fault energies for model alloys with compositions similar to AISI-316L austenitic steels. Moreover, the potential provides the stability of the fcc phase for Fe-10Ni-20Cr under large shear strains (5\%) in the temperature range from $0 \mathrm{~K}$ to $900 \mathrm{~K}$.

The potentials proposed in reference [34] were used to describe the $\mathrm{Fe}-\mathrm{He}$ and $\mathrm{Cr}-\mathrm{He}$ interactions. Based on the pair potential formalism, these potentials were fitted in order to correctly reproduce, with respect to DFT results, the migration energies of helium in Fe and $\mathrm{Cr}$ and the formation energy of the substitutional and interstitial helium in tetrahedral and 
octahedral sites in $\mathrm{Fe}$ and $\mathrm{Cr}$.

The potential describing the $\mathrm{Ni}-\mathrm{He}$ interaction was fitted to a set of $\mathrm{Ni}-\mathrm{He}$ interaction energies obtained by Melius [35] using the Hartree-Fock approximation. The form of this potential is given below:

$$
V(r)=\left\{\begin{array}{cc}
\left(A+\frac{B}{r}+\frac{C}{r^{2}}\right) e^{-D r} & r<r_{i} \\
P_{3} r^{3}+P_{2} r^{2}+P_{1} r+P_{0} & r_{i} \leq r \leq r_{c} \\
0 & r>r_{c}
\end{array}\right.
$$

The potential parameters, together with the cutoff radii, are given in Table 1.

To our knowledge, two other Ni-He potentials have been recently proposed: one by Zhang et al. [36] and the other one by Torres et al. [37]. These two potentials are both able to well reproduce, with respect to DFT results, the incorporation energies of helium in substitution and interstitial tetrahedral and octahedral sites in $\mathrm{Ni}$ and the helium migration barrier between two tetrahedral sites. The Zhang potential coincides with our potential for $r>0.1 \mathrm{~nm}$ but tends to be less repulsive for $r<0.1 \mathrm{~nm}$, while the Torres potential is much less repulsive. In a series of test calculations, the Ni-He potential used in the present work (referred to as Melius potential) was compared with the most different of the two previously mentioned potentials, the Torres potential. These test calculations showed practically no difference (see Table 2) between the bubble radii $\left(R_{B}\right)$, average densities $(\rho)$ and pressures in the bubble $\left(P_{B}\right)$ calculated with the two Ni-He potentials.

A comparison [38] between two He-He potentials, the Ross-Young (RY) potential [39] and the Aziz potential [40], showed that the former is able to better reproduce the $P(\rho)$ curve, with respect to DFT and experimental results (at least at $\sim 300 \mathrm{~K}$ ), while the latter shows a 
better agreement with the experimental data on melting curves. The RY potential also predicts an fcc-bcc-fluid triple point at $P=12.5 \mathrm{GPa}$ and $T=250 \mathrm{~K}$, while no such point was observed with the Aziz potential. Indeed, a triple point was experimentally detected at $P=11 \mathrm{GPa}$ and $T=285 \mathrm{~K}$ [41], as predicted by the RY potential, albeit of a different (fcc-hcp-fluid) type.

Given the relatively good description of the $P(\rho)$ curve by the RY potential and the fact that the $T-P$ range of the calculations from the present work do not include the helium melting curve (the helium is firmly in the liquid phase [38]), the RY potential was considered well suited to describe the He-He interactions in our MD simulations.

\section{Results and discussion}

As shown on Figure 1, the M-He interactions dominating at the helium/steel interface are more repulsive than the $\mathrm{He}-\mathrm{He}$ ones, which are predominant in the central region of the bubble. This imbalance between the interactions at the interface and in the bubble center induces a He desorption from the helium/steel interface in order to equalize its chemical potential throughout the bubble. Thus, as observed on the density profiles calculated after the MD relaxation of the helium/steel systems, the bubble exhibits a central bulk-like region and a low density region, or even a gap (Figure 2), close to the bubble surface $\left(r=R_{B}\right)$. The occurrence of a gap at the interface has already been observed for helium bubbles in bcc Fe and $\mathrm{FeCr}$ alloys $[15,17]$ and this gap has been shown to decrease and even disappear at high helium densities [15].

As a result of the non-uniform He density profile, the first radial distribution function peaks of the helium in the bubble are more intense and slightly shifted towards lower He-He distances compared to bulk helium at the same average density $\rho$ (Figure 3). 
The repulsive helium/steel interactions also translate in higher pressures in the bubble $\left(P_{B}\right)$ as compared to bulk helium $\left(P_{P}\right)$ at the same temperature $T$ and average density $\rho$ :

$$
P_{B}(\rho, T)>P_{P}(\rho, T)
$$

Figure 4 illustrates this inequality for systems at $500 \mathrm{~K}$ and different densities. This figure also shows that the discrepancies between the pressure in the bubble and the bulk helium one diminish as the bubble radius increases. The helium behaves as if it occupied in the bubble a volume $V_{c}$ (confinement volume) smaller than the bubble volume $V_{B}$. To this confinement volume one can associate a confinement density $\left(\rho_{c}\right)$ that is higher than the average helium density in the bubble $(\rho)$. The confinement density can be considered as the virtual value of the bulk helium density for which the pressure in the bubble equals the bulk helium one:

$$
P_{B}(\rho, T)=P_{P}\left(\rho_{c}, T\right)
$$

If $P_{P}\left(\rho_{c}, T\right)$ from the equation 3 is expressed using the bulk helium virial EOS in terms of density, one obtains an EOS for the helium in the bubble:

$$
P_{B}(\rho, T)=\rho_{c} k T+\sum_{i \geq 1} F_{i}(T) \rho_{c}^{i+1}
$$

The $F_{i}(T)$ coefficients are functions of temperature while $\rho_{c}$, the confinement density, is a function of the average helium density in the bubble, $\rho$.

In order to find a simple form for the $\rho_{c}(\rho)$ function, one makes the hypothesis (in agreement with experimental findings $[7,10])$ that the helium bubbles in steel have a spherical shape with the helium occupying only a central (spherical) zone of the bubble. Within this hypothesis, the $\rho_{d} \rho$ ratio can be written as shown below: 


$$
\frac{\rho_{c}}{\rho}=\frac{V_{B}}{V_{c}}=\frac{R_{B}^{3}}{\left(R_{B}-\Delta R\right)^{3}}=C\left(R_{B}\right)
$$

In equation $5, R_{B}$ is the bubble radius and $\Delta R$ is a correction term that accounts for the repulsive effect of the helium/steel interface. It follows from this equation that, as for the pressure, the discrepancies between $\rho_{c}$ and $\rho$ diminish with increasing bubble radius, vanishing for an infinite radius.

Expressing $\rho_{c}$ as a function of $\rho$ from equation 5 and developing the equation 4 up to the third coefficient $(i=3)$, one obtains the following relationship:

$$
P_{B}(\rho, T)=\rho C\left(R_{B}\right) k T+\sum_{i=1}^{3}\left(A_{i} T+B_{i}\right) \rho^{i+1} C^{i+1}\left(R_{B}\right)
$$

where the $F_{i}(T)$ coefficients from equation 4 are expressed as affine functions of $T$. The choice of an affine function is justified by the narrow temperature range $(T=500-700 \mathrm{~K})$ analysed in the present study. For a larger temperature range, higher order polynomial functions could be necessary. The $A_{i}$ and $B_{i}$ parameters (Table 3) were obtained by fitting to a set of MD data points calculated for the bulk helium using the two-phase method described in reference [38] and the He-He interatomic potential introduced in Section 2.3.

The value of the $\Delta R$ correction term was computed by fitting to the MD pressure data obtained in the present work. The fitting procedure involved the introduction of a penalty function $S(\Delta R)$ :

$$
S(\Delta R)=\frac{1}{N_{P}} \sum_{i=1}^{N_{P}}\left[P_{i}^{E O S}(\Delta R)-P_{i}^{M D}\right]^{2}
$$

$P_{i}^{E O S}(\Delta R)$ is the pressure in the bubble given by equation 6 and $P_{i}^{M D}$ is the pressure in the bubble calculated through MD. $N_{P}$ represents the number of molecular dynamics data points. 
The value of $\Delta R$ that minimizes the penalty function is taken as the correction parameter in equation 6 . For both MD data sets, at $500 \mathrm{~K}$ and $700 \mathrm{~K}$, one obtained the same $\Delta R$ value of $0.0549 \mathrm{~nm}(0.549 \AA)$. This shows that the temperature has a negligible impact on the $\Delta R$ value.

This kind of correction was already successfully applied by other authors in the case of helium bubbles in bcc iron [22]. These authors used the $\Delta R$ correction to adapt a bulk helium hard sphere equation of state to the bubble case. Fitting on MD results they obtained on a large temperature range (from $300 \mathrm{~K}$ to $1000 \mathrm{~K}$ ), these authors found for $\Delta R$ a value very similar to the present one: $0.057 \mathrm{~nm}$.

Figure 5 gives a comparison between the pressure in the bubble calculated using the equation 6 and the corresponding MD pressure values. The uncorrected $\operatorname{EOS}(\triangle R=0 \mathrm{~nm})$ always underestimates the pressure in the bubble. Introducing the $\Delta R$ correction allows to considerably improve the EOS results.

In order to quantify the deviation of the pressure in the bubble from the bulk helium one at the same density and temperature, one defined a relative deviation function, $\operatorname{Dev}\left(R_{B}\right) \%$, as follows:

$$
\operatorname{Dev}\left(R_{B}\right) \%=\frac{P^{E O S}\left(R_{B}\right)-P_{P}}{P_{P}} \times 100
$$

$P^{E O S}\left(R_{B}\right)$ is the helium pressure in a $R_{B}$ radius bubble calculated with the corrected EOS (equation 6) and $P_{P}$ is the bulk helium pressure at the same temperature and density as in the bubble. Figure 6 represents the $\operatorname{Dev}\left(R_{B}\right) \%$ function calculated at $600 \mathrm{~K}$ (in the middle of the analysed temperature range) for three density values covering all the analysed density range: $20 \mathrm{~nm}^{-3}, 60 \mathrm{~nm}^{-3}$ and $100 \mathrm{~nm}^{-3}$. The relative deviation decreases with $R_{B}$, this decrease being 
steeper at lower densities. In general, for $R_{B}>5 \mathrm{~nm}$ the relative deviation is less than $10 \%$ and for $R_{B}>10 \mathrm{~nm}$ it becomes less than $5 \%$. Thus, for bubble radii in excess of $10 \mathrm{~nm}$ a bulk EOS can be safely used to describe the state of helium in the bubble.

\section{Conclusions}

The study presented here showed that the steel surface has a strong impact on the helium behaviour in the bubble, that is, on its equation of state. Thus, in order to correctly describe the helium state in a bubble, a different EOS from that of the bulk helium should be used. In the present work, a bulk helium virial EOS was altered by introducing a spherical confinement volume inside the bubble that accounts for the (repulsive) effects of the surface.

An analysis of the relative deviation of the pressure in the bubble from the bulk helium one at the same density and temperature showed that, for bubble radii in excess of $10 \mathrm{~nm}$, a bulk helium EOS would become appropriate to describe the helium in the bubble.

The helium EOS in the bubble provided by this study can be further used in the kinetic equations describing the growth rate of the helium filled cavities [42] that form in the austenitic steels under neutron irradiation.

\section{Acknowledgments}

All the calculations presented in the article were performed using the IRSN computing resources. 


\section{References}

[1] R.L. Plaut, C. Herrera, D.M. Escriba, P.R. Rios, A.F. Padilha, Materials Research 10 (2007) 453-460.

[2] F.A. Garner, Comprehensive Nucl. Mater. 4 (2012) 33-95.

[3] H. Trinkaus, B.N. Singh, J. Nucl. Mater. 323 (2003) 229-242.

[4] R.E. Stoller, J. Nucl. Mater. 174 (1990) 289-310.

[5] B.A. Kalin, S.N. Korshunov, I.I. Chernov, A.V. Markin, I.V. Reutov, V.N. Chernikov, A.P. Zakharov, V.M. Gureev, M.I. Guseva, J. Nucl. Mater. 161 (1989) 228-238.

[6] E.H. Lee, A.F. Rowcliffe, L.K. Mansur, J. Nucl. Mater. 103-104 (1981) 1475-1480.

[7] E.H. Lee, J.D. Hunn, T.S. Byun, L.K. Mansur, J. Nucl. Mater. 280 (2000) 18-24.

[8] M. Snykers, E. Ruedl, J. Nucl. Mater. 103-104 (1981) 1075-1078.

[9] T. Sawai, K. Fukai, S. Jitsukawa, H. Otsu, A. Hishimuma, J. Nucl. Mater. 212-215 (1994) 453-457.

[10] I.I. Chernov, A.N. Kalashnikov, B.A. Kalin, S.Y. Binyukova, J. Nucl. Mater. 323 (2003) $341-345$

[11] T. Lauritzen, A. Withop, U.E. Wolff, Nuclear Engineering and Design 9 (1969) 265-268.

[12] S. Jublot-Leclerc, M.-L. Lescoat, F. Fortuna, L. Legras, X. Li, A. Gentils, J. Nucl. Mater. 466 (2015) 646-652.

[13] B. Viswanathan, J. Phys.: Condens. Matter 1 (1989) SA71-SA76. 
[14] M. Samaras, Mater. Today 12 (2009) 46-53.

[15] S.M. Hafez Haghighat, G. Lucas, R. Schäublin, EPL 85 (2009) 60008: 1-5.

[16] S.H. Guo, B.E. Zhu, W.C. Liu, Z.Y. Pan, Y.X. Wang, Nucl. Instr. Meth. Phys. Res. B 267 (2009) 3278-3281.

[17] A. Caro, J. Hetherly, A. Stukowski, M. Caro, E. Martinez, S. Srivilliputhur, L. ZepedaRuiz, M. Nastasi, J. Nucl. Mater. 418 (2011) 261-268.

[18] J. Hetherly, E. Martinez, M. Nastasi, A. Caro, J. Nucl. Mater. 419 (2011) 201-207.

[19] T. Jourdan, J.-P. Crocombette, J. Nucl. Mater. 418 (2011) 98-105.

[20] D. Stewart, Y. Osetskiy, R. Stoller, J. Nucl. Mater. 417 (2011) 1110-1114.

[21] A. Caro, D. Schwen, E. Martinez, Appl. Phys. Lett. 103 (2013) 213115: 1-4.

[22] R.E. Stoller, Y.N. Osetsky, J. Nucl. Mater. 455 (2014) 258-262.

[23] A. Caro, D. Schwen, J. Hetherly, E. Martinez, Acta Materialia 89 (2015) 14-21.

[24] G. Bonny, D. Terentyev, R.C. Pasianot, S. Poncé, A. Bakaev, Modelling Simul. Mater. Sci. Eng. 19 (2011) 085008: 1-14.

[25] J.B. Piochaud, Ph.D. Thesis, Université des Sciences et Technologies de Lille, 2013.

[26] S. Fréchard, M. Walls, M. Kociak, J.P. Chevalier, J. Henry, D. Gorse, J. Nucl. Mater. 393 (2009) 102-107.

[27] Y. Wu, R.G. Odette, T. Yamamoto, J. Ciston, P. Hosemann, Fusion Reactor Materials Program DOE/ER-0313/54 54 (2013) 173-179. 
[28] L.M. Caspers, R.H.J. Fastenau, A. van Veen, W.F.W.M van Heugten, Phys. Stat. Sol. A 46 (1978) 541-546.

[29] H. Trinkaus, G. Wolfer, J. Nucl. Mater. 122 \& 123 (1984) 552-557.

[30] G.W. Greenwood, A.J.E. Foreman, D.E. Rimmer, J. Nucl. Mater. 4 (1959) 305-324.

[31] A.R. Leach, Molecular Modelling Principles and Applications, Pearson Education Limited, Harlow, 2001.

[32] S. Plimpton, J. Comput. Phys. 117 (1995) 1-19.

[33] A. Jelea, R.J.-M. Pellenq, F. Ribeiro, J. Nucl. Mater. 444 (2014) 153-160.

[34] D. Terentyev, N. Juslin, K. Nordlund, N. Sandberg, J. of Appl. Phys. 105 (2009) 103509: $1-12$.

[35] C.F. Melius, C.L. Bisson, W.D. Wilson, Phys. Rev. B 18 (1978) 1647-1657.

[36] W. Zhang, C. Wang, H. Gong, Z. Xu, C. Ren, J. Dai, P. Huai, Z. Zhu, H. Deng, W. Hu, J. Nucl. Mater. 472 (2016) 105-109.

[37] E. Torres, J. Pencer, D.D. Radford, J. Nucl. Mater. 479 (2016) 240-248.

[38] L. Koci, R. Ahuja, A.B. Belonoshko, B. Johansson, J. Phys.: Condens. Matter. 19 (2007) 016206: 1-9.

[39] M. Ross, D.A. Young, Phys. Lett. A 118 (1986) 463-466.

[40] R.A. Aziz, V.P.S. Nain, J.C. Carley, W.L. Taylor, G.T. McConville, J. Chem. Phys. 70 (1979) 4330-4342. 
[41] P. Loubeyre, R. LeToullec, J.P. Pinceaux, H.K. Mao, J. Hu, R.J. Hemley, Phys. Rev. Lett. 71 (1993) 2272-2275.

[42] L.K. Mansur, Nucl. Tech. 40 (1978) 5-34. 


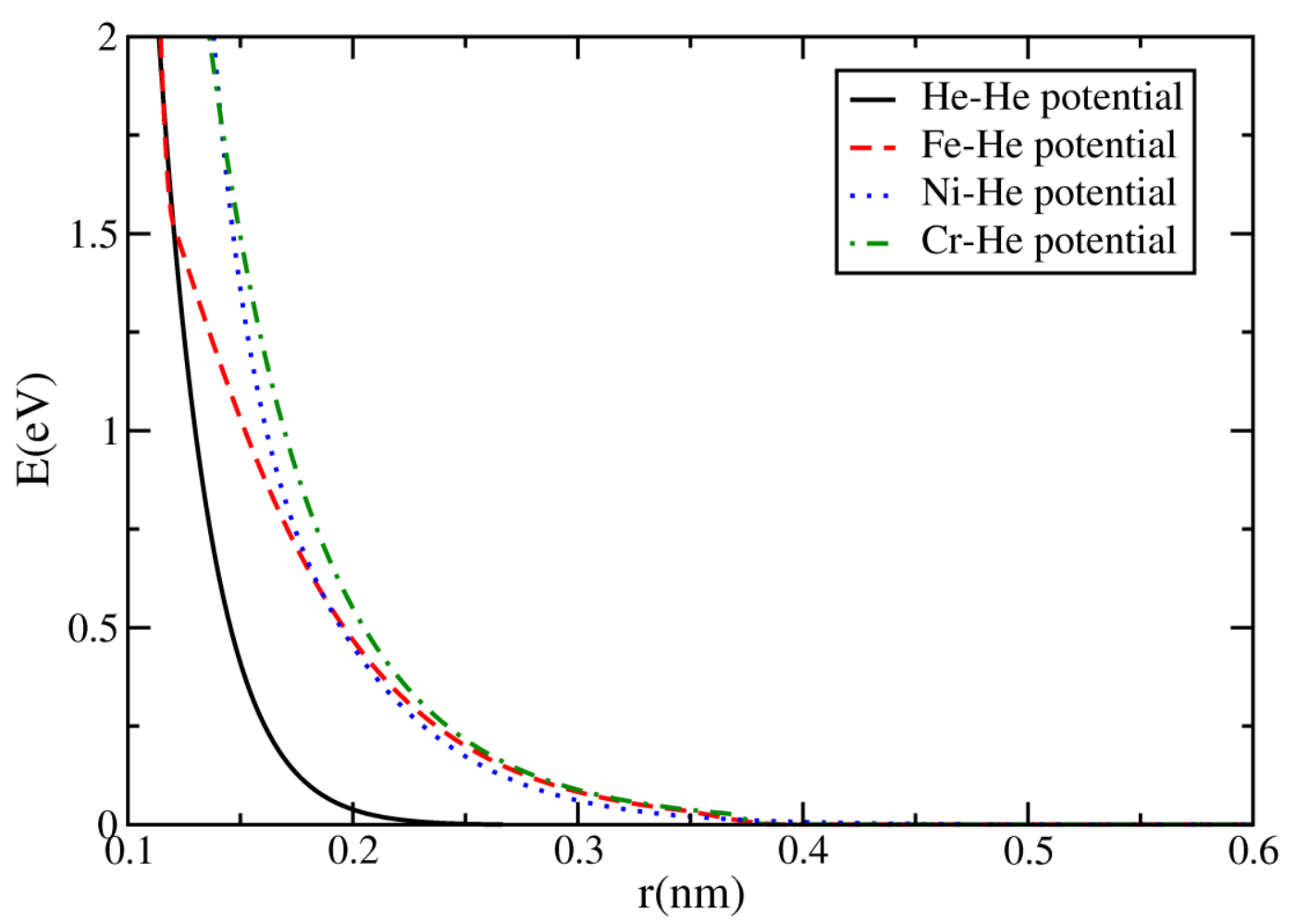

Fig. 1 


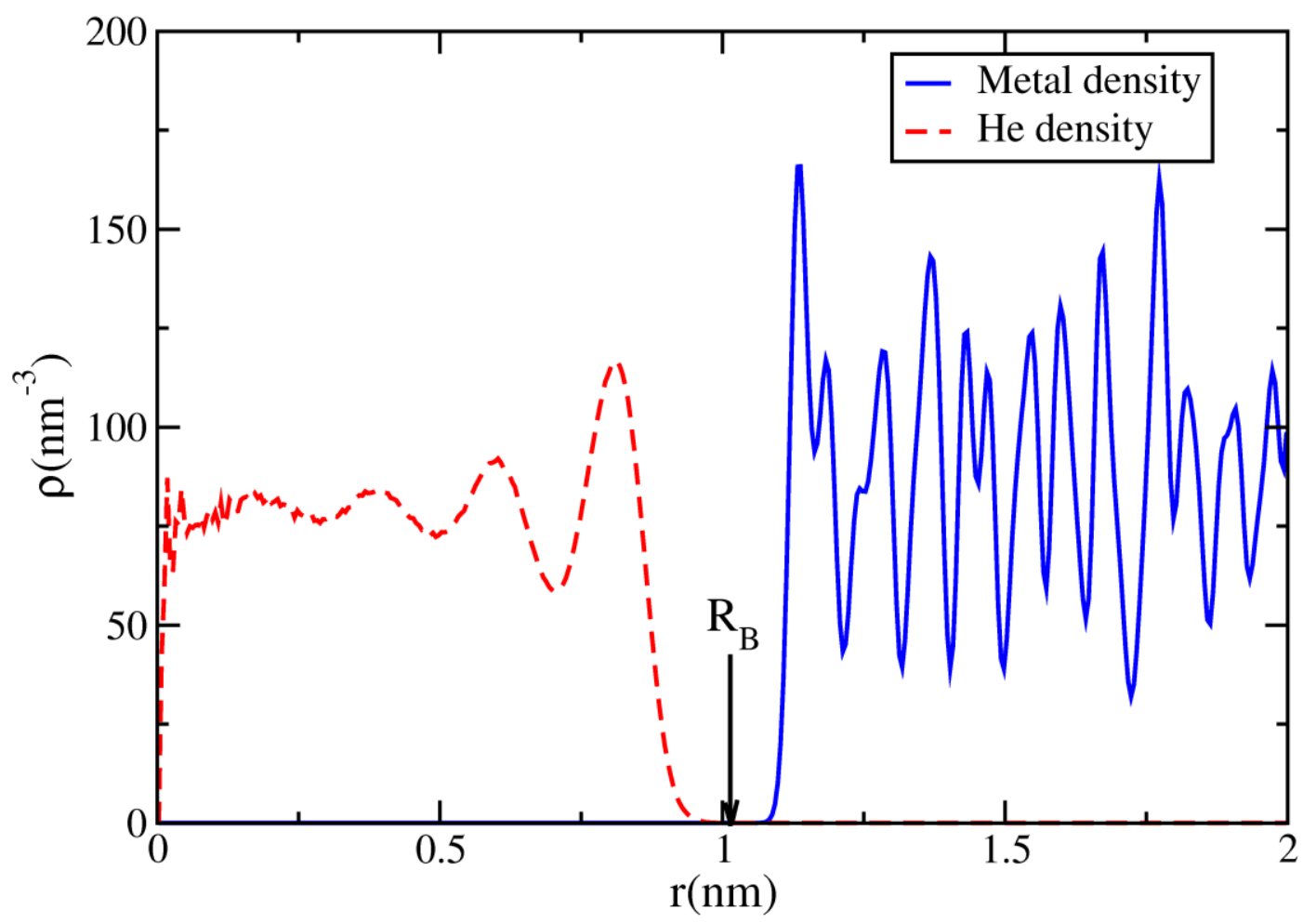

Fig. 2 


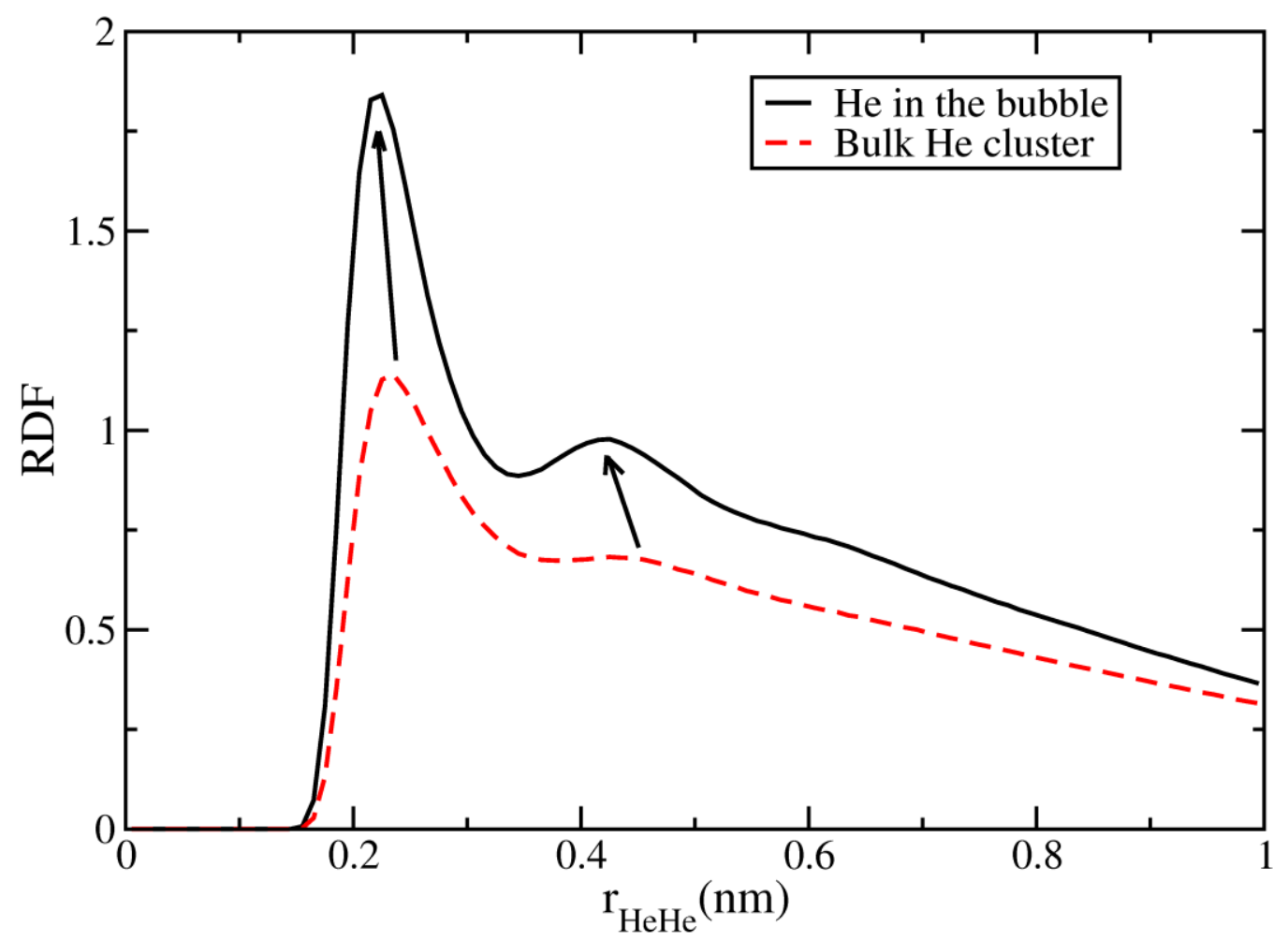

Fig. 3 


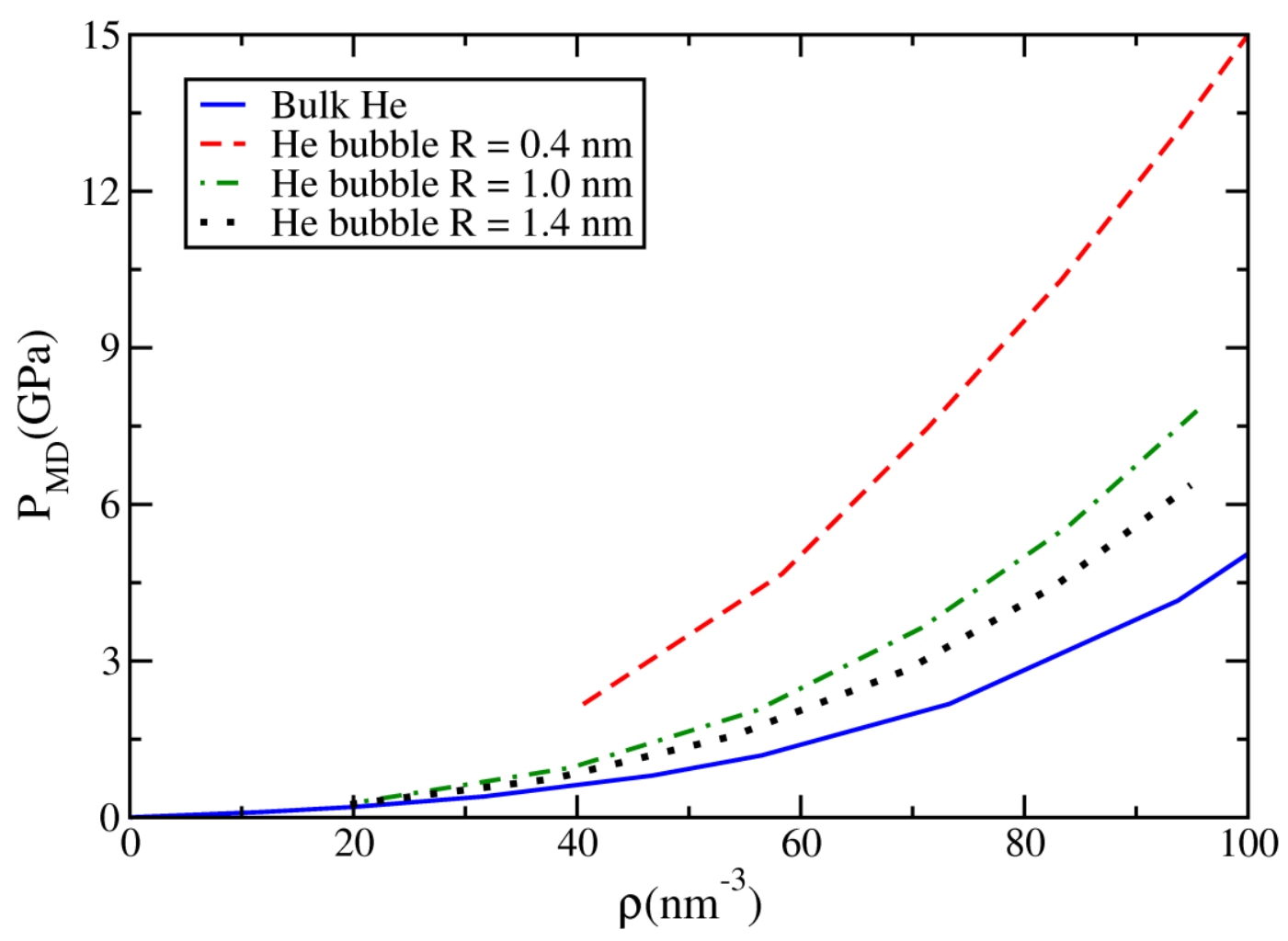

Fig. 4 


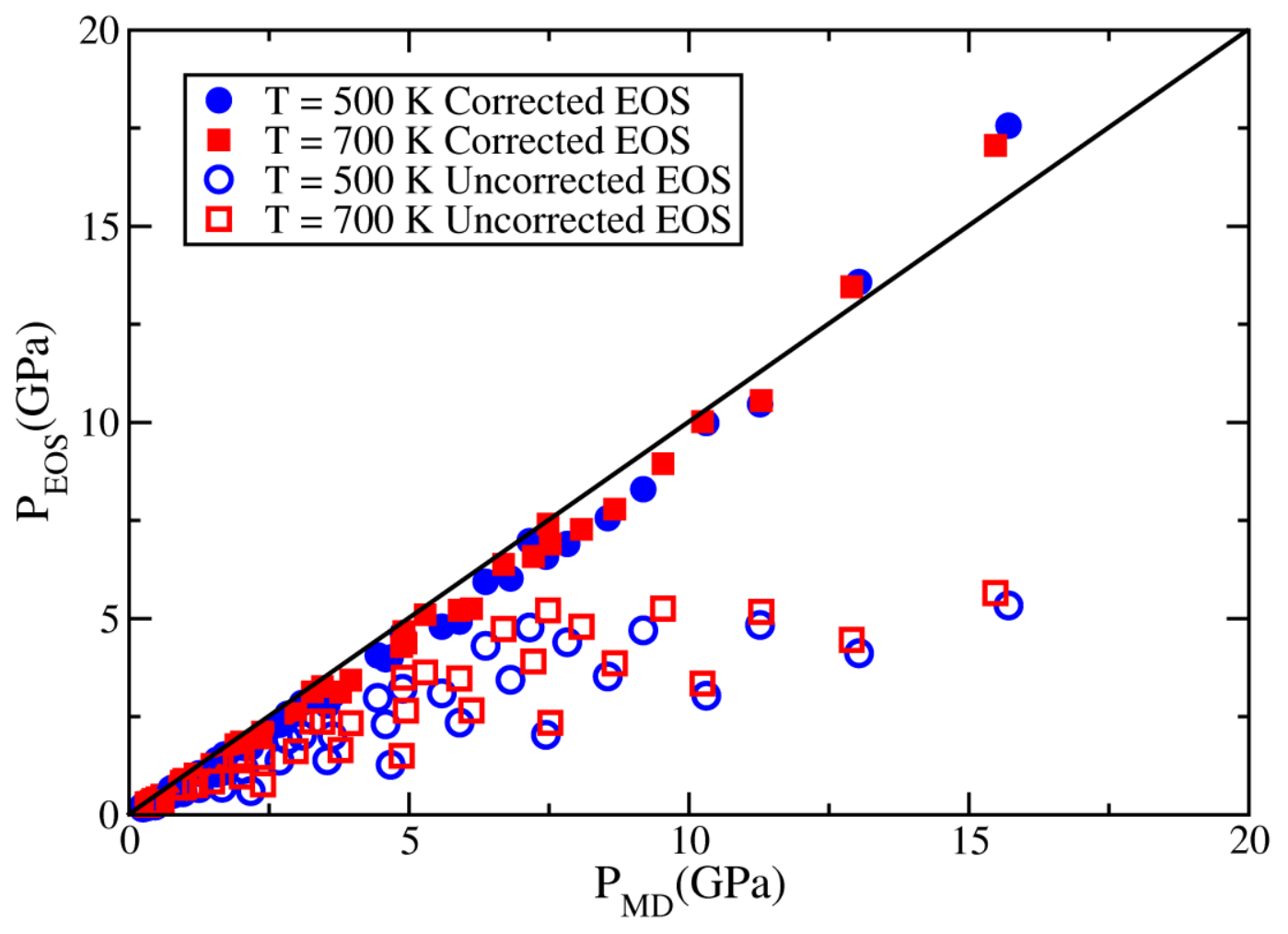

Fig. 5 


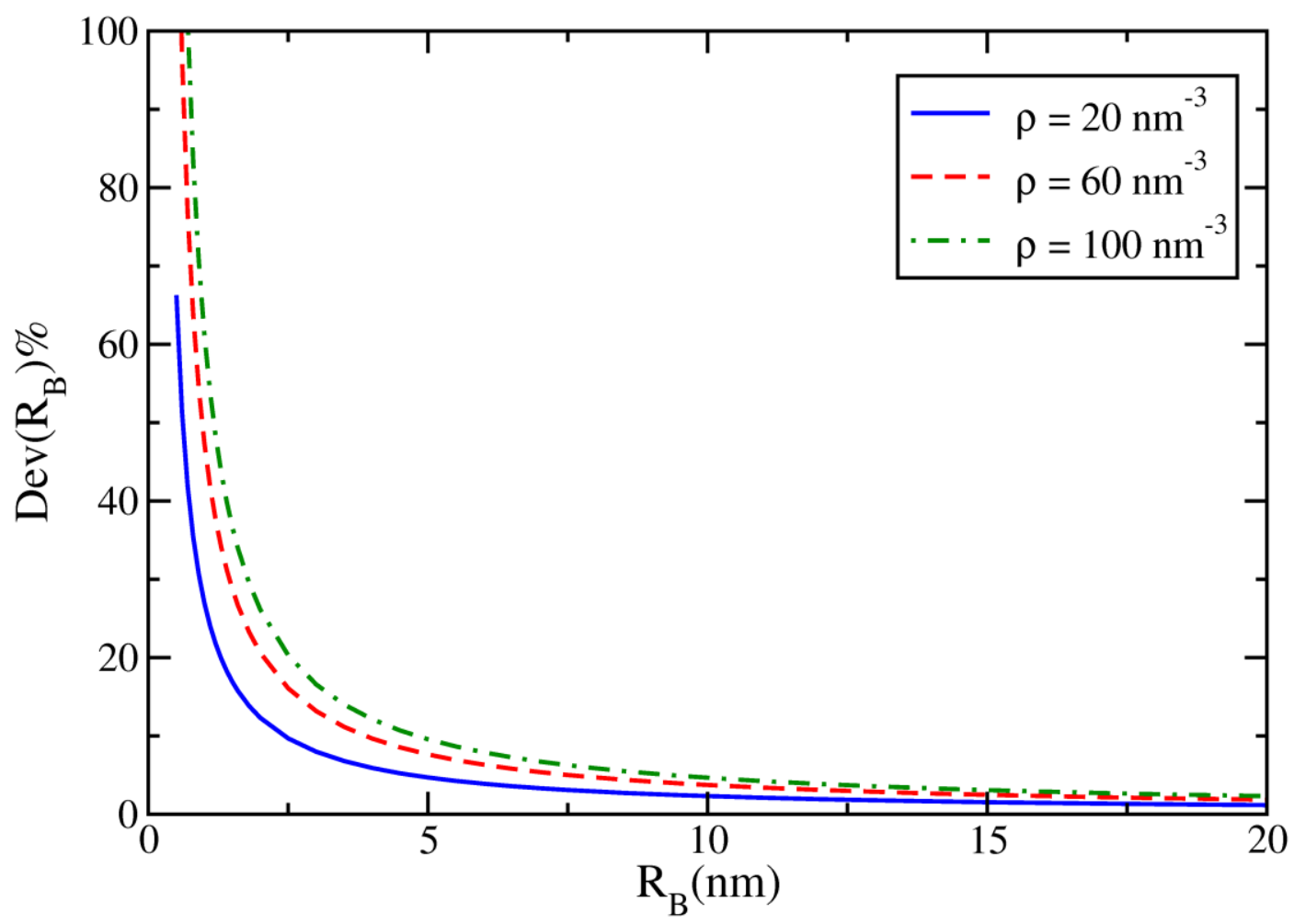

Fig. 6 


\section{Figure captions}

Fig. 1. Comparison between the $\mathrm{He}-\mathrm{He}$ and $\mathrm{M}-\mathrm{He}(\mathrm{M}=\mathrm{Fe}, \mathrm{Ni}, \mathrm{Cr})$ potentials.

Fig. 2. Example of helium distribution in a bubble in fcc FeNiCr. The radial density profile was calculated at $500 \mathrm{~K}$ for a $1 \mathrm{~nm}$ bubble. The average He density in the bubble is $56 \mathrm{~nm}^{-3}$.

Fig. 3. Comparison between the radial distribution function (RDF) of the helium in a $1 \mathrm{~nm}$ bubble in fcc FeNiCr $\left(T=500 \mathrm{~K}, \rho=56 \mathrm{~nm}^{-3}\right)$ and the RDF of the bulk helium at the same density and temperature. The RDF for bulk helium was calculated for a cluster of atoms found in a $1 \mathrm{~nm}$ radius sphere.

Fig. 4. Comparison between the $P(\rho)$ curves corresponding to the helium in bubbles with various radii and that of the bulk helium at $500 \mathrm{~K}$.

Fig. 5. Comparison between the pressures in the bubble calculated using corrected and uncorrected virial EOS and the corresponding MD results.

Fig. 6. The relative deviation of the pressure in the bubble from the bulk helium one at the same density and temperature represented as a function of the bubble radius $R_{B}$. 


\section{Table 1}

Parameters for the Ni-He potential used in the present work. The unit for energy is the electronvolt $(\mathrm{eV})$ and the unit for distance is the angstrom $(\AA)$.

\begin{tabular}{ccccc}
\hline$A(\mathrm{eV})$ & $B(\mathrm{eV} \cdot \AA)$ & $C\left(\mathrm{eV} \cdot \AA^{2}\right)$ & $D\left(\AA^{-1}\right)$ & $r_{i}(\AA)$ \\
\hline 349.732 & -917.546 & 727.518 & 2.54144 & 4.0 \\
\hline$P_{0}(\mathrm{eV})$ & $P_{l}\left(\mathrm{eV} \cdot \AA^{-1}\right)$ & $P_{2}\left(\mathrm{eV} \cdot \AA^{-2}\right)$ & $P_{3}\left(\mathrm{eV} \cdot \AA^{-3}\right)$ & $r_{c}(\AA)$ \\
\hline $3.71640 \mathrm{E}-1$ & $-2.01704 \mathrm{E}-1$ & $3.60846 \mathrm{E}-2$ & $-2.12190 \mathrm{E}-3$ & 5.0 \\
\hline
\end{tabular}


Table 2

Comparison between the bubble radii $\left(R_{B}\right)$, average densities $(\rho)$ and pressures in the bubble $\left(P_{B}\right)$ predicted at $700 \mathrm{~K}$ using two different $\mathrm{Ni}-\mathrm{He}$ potentials: the Melius potential $(\mathrm{M})$ [35] and Torres potential $(\mathrm{T})$ [37]. $\mathrm{R}_{\mathrm{C}}$ and $\mathrm{NHe}$ stand for the cavity radius before MD relaxation and the number of helium atoms in the bubble, respectively.

\begin{tabular}{cccccc}
\hline Ni-He potential & $\mathrm{R}_{\mathrm{C}}(\mathrm{nm})$ & $\mathrm{NHe}$ & $R_{B}(\mathrm{~nm})$ & $\rho\left(\mathrm{nm}^{-3}\right)$ & $P_{B}(\mathrm{GPa})$ \\
\hline $\mathrm{M}$ & 0.5 & 38 & 0.45 & 99.0 & 15.47 \\
$\mathrm{~T}$ & 0.5 & 38 & 0.45 & 99.0 & 15.34 \\
$\mathrm{M}$ & 1.1 & 490 & 1.08 & 93.0 & 8.08 \\
$\mathrm{~T}$ & 1.1 & 490 & 1.08 & 93.0 & 8.02 \\
$\mathrm{M}$ & 1.5 & 1266 & 1.48 & 92.5 & 6.69 \\
$\mathrm{~T}$ & 1.5 & 1266 & 1.48 & 92.5 & 6.64 \\
\hline
\end{tabular}


Table 3

Parameters used in Equation 6. In this equation, the unit for the pressure is the GPa and the unit for the density is the $\AA^{-3}\left(1 \AA^{-3}=1000 \mathrm{~nm}^{-3}\right)$. As a consequence, the parameters are expressed using the following units: $1 \mathrm{GPa} \cdot \AA^{3 i+3} \cdot \mathrm{K}^{-1}$ for $A_{i}, 1 \mathrm{GPa} \cdot \AA^{3 \mathrm{i}+3}$ for $B_{i}$ and 1 $\mathrm{GPa} \cdot \AA^{3} \cdot \mathrm{K}^{-1}$ for $k$, the Boltzmann constant.

\begin{tabular}{ccccccc}
\hline$A_{1}$ & $B_{1}$ & $A_{2}$ & $B_{2}$ & $A_{3}$ & $B_{3}$ & $k$ \\
\hline 0.19103 & -1.5031 & 1.4263 & 1215.08 & -6.5035 & 17534.2 & 0.0138066 \\
\hline
\end{tabular}

